\title{
12 Mode, MIMO-Free OAM Transmission
}

Ingerslev, Kasper; Gregg, Patrick; Galili, Michael; Da Ros, Francesco; Hu, Hao; Bao, Fangdi; Usuga Castaneda, Mario A.; Kristensen, Poul; Rubano, Andrea; Marrucci, Lorenzo

Total number of authors:

14

Published in:

Optical Fiber Communication Conference 2017

Link to article, DOI:

10.1364/OFC.2017.M2D.1

Publication date:

2017

Document Version

Peer reviewed version

Link back to DTU Orbit

Citation $(A P A)$ :

Ingerslev, K., Gregg, P., Galili, M., Da Ros, F., Hu, H., Bao, F., Usuga Castaneda, M. A., Kristensen, P., Rubano, A., Marrucci, L., Ramachandran, S., Rottwitt, K., Morioka, T., \& Oxenløwe, L. K. (2017). 12 Mode, MIMO-Free OAM Transmission. In Optical Fiber Communication Conference 2017 [M2D.1] Optical Society of America (OSA). https://doi.org/10.1364/OFC.2017.M2D.1

\section{General rights}

Copyright and moral rights for the publications made accessible in the public portal are retained by the authors and/or other copyright owners and it is a condition of accessing publications that users recognise and abide by the legal requirements associated with these rights.

- Users may download and print one copy of any publication from the public portal for the purpose of private study or research.

- You may not further distribute the material or use it for any profit-making activity or commercial gain

- You may freely distribute the URL identifying the publication in the public portal 


\title{
12 Mode, MIMO-Free OAM Transmission
}

\author{
Kasper Ingerslev ${ }^{1}$, Patrick Gregg ${ }^{2}$, Michael Galili ${ }^{1}$, Francesco Da $\operatorname{Ros}^{1}$, Hao Hu${ }^{1}$, Fangdi Bao ${ }^{1}$, Mario A. \\ Usuga Castaneda $^{1}$, Poul Kristensen ${ }^{3}$, Andrea Rubano ${ }^{4}$, Lorenzo Marrucci ${ }^{4}$, Siddharth Ramachandran ${ }^{2}$, \\ Karsten Rottwitt ${ }^{1}$ Toshio Morioka ${ }^{1}$ and Leif Katsuo Oxenløwe ${ }^{1}$ \\ ${ }^{I}$ DTU Fotonik, Department of Photonics Engineering, Technical University of Denmark, DK-2800, Lyngby, Denmark \\ ${ }^{2}$ Electrical and Computer Engineering Department, Boston University, 8 St Mary's St, Boston, MA, USA \\ ${ }^{3}$ OFS-Fitel, Priorparken 680, DK-2605, Broendby, Denmark \\ ${ }^{4}$ Dipartimento di Fisica, Università di Napoli Federico II, MSA, via Cintia, 80126 Napoli, Italy \\ kaing@fotonik.dtu.dk
}

\begin{abstract}
Simultaneous MIMO-free transmission of a record number (12) of orbital angular momentum modes over $1.2 \mathrm{~km}$ is demonstrated. WDM compatibility of the system is shown by using 60 WDM channels with $25 \mathrm{GHz}$ spacing and 10 GBaud QPSK.

OCIS codes: (060.2330) Fiber optics communications; (080.4865) Optical vortices; (060.4230) Multiplexing
\end{abstract}

\section{Introduction}

Orbital angular momentum (OAM) carrying modes of optical fibers have received significant attention recently for their potential use in mode division multiplexing (MDM) as independent data carriers without the use of cost and power intensive multiple-input-multiple-output (MIMO) digital signal processing. OAM modes are characterized by a helical phase profile, $\exp (i L \varphi)$, where $\varphi$ is the azimuthal coordinate and $L$ is the topological charge, and a circular polarization $\sigma^{ \pm}=\hat{x} \pm i \hat{y}$. They come in pairs with OAM and circular polarization of the same handedness (spinorbit aligned) or different handedness (spin-orbit anti-aligned). With appropriate fiber design, such modes are separated from their nearest neighbors in effective index $\left(\mathrm{n}_{\text {eff }}\right)$, and have been shown to be propagation-stable on the order of kilometers [1].

Previously, MIMO-free data transmission has been demonstrated with four OAM modes over km-lengths of suitably designed optical fibers [2,3]. Although fibers supporting a larger number of OAM states have been designed and tested [4,5], data transmission over a larger ensemble of OAM modes remains to be demonstrated.

This work investigates MDM transmission using $10 \mathrm{GBd}$ QPSK of $12 \mathrm{OAM}$ modes, i.e. the largest number of mode groups transmitted MIMO-free, over $1.2 \mathrm{~km}$. It is found that all the data channels are below the soft-decision forward error correction (SD-FEC) limit using a $25 \mathrm{GHz}$-spaced frequency comb producing $60 \mathrm{WDM}$ channels.

\section{Experimental setup}

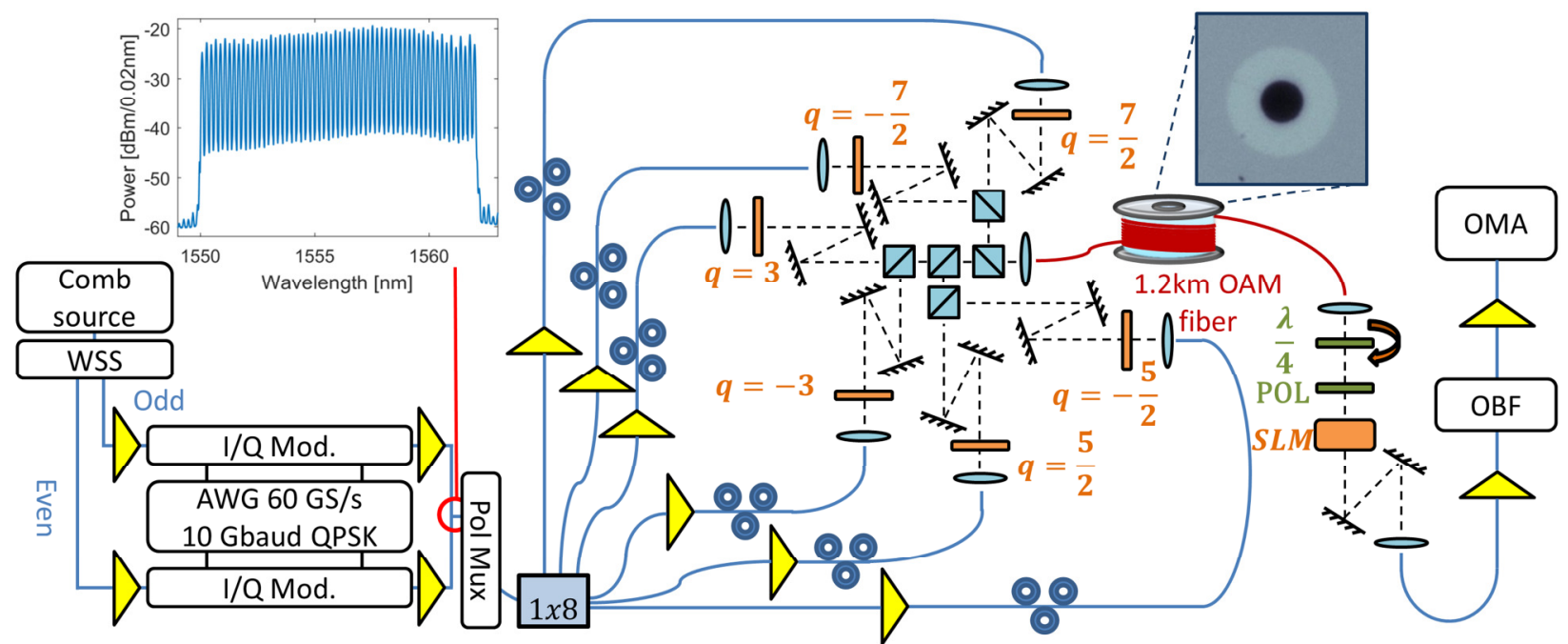

Fig. 1. Schematic of experimental setup, including the modulated spectrum (top left inset), and a microscope image (top right inset) of the OAM fiber. WSS: Wavelength Selective Switch, AWG: Arbitrary Waveform Generator, SLM: Spatial Light Modulator, OBF: Optical Bandpass Filter, OMA: Optical Modulation Analyzer.

The experimental setup is outlined in Fig.1. A frequency comb with $60 \mathrm{WDM}$ channels, spanning from $1550 \mathrm{~nm}$ to $1562 \mathrm{~nm}$ with $25 \mathrm{GHz}$ spacing, is generated by using a $1544 \mathrm{~nm} \mathrm{CW}$ source which is phase- and intensity modulated, for initial side-band generation. This is amplified and passed through two stages of highly non-linear 
fiber in order to broaden the optical spectrum further. The comb is spectrally flattened, and split into even and odd channels using a wavelength selective switch (WSS). The even and odd channels are modulated separately, with 10 GBd QPSK signals, and combined using a $3 \mathrm{~dB}$ polarization maintaining coupler. The comb is then passed through a polarization division multipexing emulator, resulting in an output signal carrying data on two orthogonal polarizations, with a differential delay of $300 \mathrm{ps}$. The signal is then split in a 1x8 coupler, two outputs are discarded, and the 6 remaining polarization-multiplexed outputs are amplified individually for use as independent data channels for the 12 OAM modes.

Each of the 6 amplified outputs of the 1x8 coupler is passed through a polarization controller and collimated. Each path is passed through a $q$-plate [6], which generates a polarization-dependent combination of OAM beams: either a combination of $+L \sigma^{+}$and $-L \sigma^{-}$in the spin-orbit aligned case, or $+L \sigma^{-}$and $-L \sigma^{+}$in the anti-aligned case, is achieved by this architecture. The linear combination of the two degenerate states created by each q-plate is adjusted, by controlling input single-mode fiber (SMF) polarization, such that degenerate state mixing in the optical fiber is "unwound." The 6 paths are combined using 5 beam-splitters, and are coupled into the OAM fiber, resulting in a total of 12 multiplexed modes; $L= \pm 5 \sigma^{ \pm}, L= \pm 5 \sigma^{\mp}, L= \pm 6 \sigma^{ \pm}, L= \pm 6 \sigma^{\mp}, L= \pm 7 \sigma^{ \pm}$and $L= \pm 7 \sigma^{\mp}$.

The signal is transmitted through $1.2 \mathrm{~km}$ of OAM fiber, and the output is collimated, and sent through a circular polarizer, comprising a quarter-wave plate and a linear polarizer. A spatial light modulator (SLM) converts the detected mode to a $L=0$ Gaussian-like field, after which the beam is coupled into a SMF. The setting of the quarter-wave plate along with the phase imparted by the SLM allow choosing the fiber mode that is projected back to a Gaussian beam, that couples the data stream to the output SMF. Thereafter, the desired WDM channel is selected using a tunable optical bandpass filter (OBF) and sent to an optical modulation analyzer (OMA), handling demodulation, and error counting. Since the aforementioned setup comprises a series of 3-dB beam combiners, input losses for the 12 OAM modes are not the same. Hence, the 6 outputs of the $1 \times 8$ coupler prior to the free-space incoupling setup have individual amplifiers set for equalizing the detected power in each mode.

The system is aligned using a pulsed source and measuring in-situ the temporal impulse response of each arm independently. The polarization of each arm is aligned by temporarily removing one input to the polarization mux, and adjusting polarization such that the output projection into one degenerate state is minimized across the frequency comb. It is observed that it is not always possible to achieve a strong suppression of degenerate states across the entire comb, which may be due either to imperfect input coupling, or to in-fiber mode coupling [7].

\section{Results and discussion}
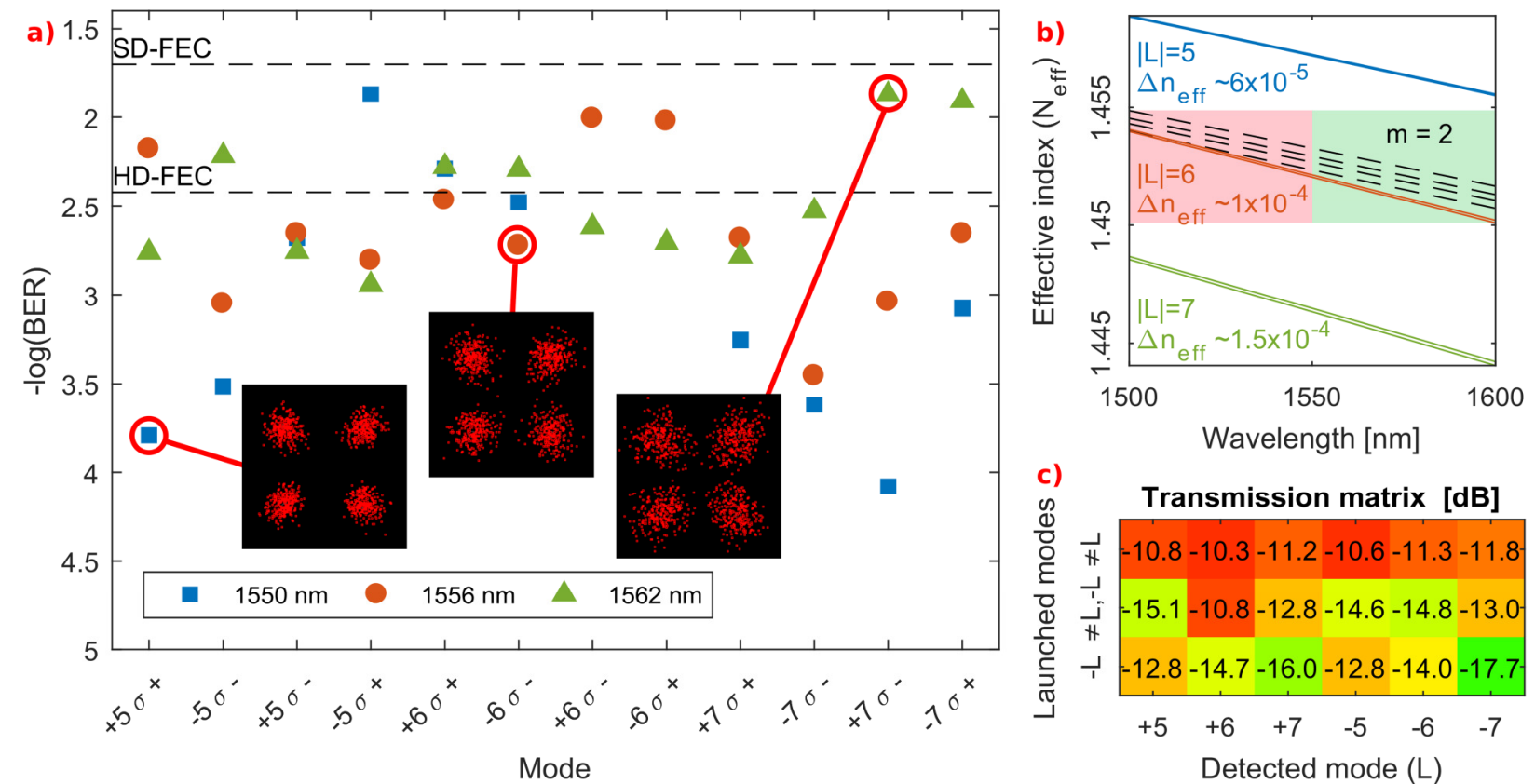

Fig. 2. a) Measured BERs with constellation diagrams (inset). b) Simulated $n_{\text {eff }}$ as a function of wavelength, the red area denotes the bandwidth where the $L=6$ anti-aligned modes have strong MPI. c) System transmission matrix - each column corresponds to an output projection setting, while the rows correspond to modes launched. Each column is normalized to $0 \mathrm{~dB}$ in the desired mode with entries denoting crosstalk in $\mathrm{dB}$. $-L$ indicates crosstalk from in-fiber nearest neighbors $, \neq L,-L$ indicates contributions from other mode orders, and $\neq L$ indicates total crosstalk. 
Bit error rates (BERs) are measured at 3 different wavelengths: $1550 \mathrm{~nm}, 1556 \mathrm{~nm}$, and $1562 \mathrm{~nm}$, corresponding to the comb's shortest, center, and longest wavelength. The measurements are performed with the fiber fully loaded with 60 WDM channels and 12 modes. The measured BERs are seen in Fig. 2 a). All 34 measurements are below the SD-FEC limit, while 24 are below HD-FEC. The $L= \pm 6 \sigma^{\mp}$ (anti-aligned) modes at $1550 \mathrm{~nm}$ experience a mode crossing (accidental degeneracy) with undesired modes of radial mode order, $m=2$ in this fiber, as shown in Fig. 2 b). This mode crossing can lead to significant multipath interference (MPI) and degradation of the signal, and so while all 12 modes were detected at the other two wavelengths, only 10 modes were detected at $1550 \mathrm{~nm}$. By design, this fiber should have experienced no mode crossing for any of the 12 modes. It is thus conceivable that future iterations of fabricating this fiber would yield transmission in all 12 modes across the C-band.

System performance is limited by intermodal crosstalk, which was measured to be between $-10.3 \mathrm{~dB}$ and $-11.8 \mathrm{~dB}$, depending on the mode. This crosstalk has two contributions: in-fiber coupling, and misalignments in the mux/demux. The system transmission matrix was measured to determine the sources of crosstalk, and is shown in Fig. 2 c). Each column corresponds to an output projection setting, while each row indicates which modes are launched and thus contribute to crosstalk at the receiver. It was found that the strongest parasitic contribution for the $|L|=5$ modes is in-fiber crosstalk between the $|L|=5$ spin-orbit aligned and anti-aligned modes $(-12.8 \mathrm{~dB})$, because this mode group has the smallest $n_{\text {eff }}$ splitting of $6 \times 10^{-5} .|L|=6$ and $|L|=7$ are mainly limited by crosstalk from modes of different $|L|$. Since the $\mathrm{n}_{\text {eff }}$ splitting between $L$ and $L \pm 1$ is an order of magnitude larger than between modes with the same $|L|$ (see Fig. 2 b), in-fiber mode coupling between adjacent OAM mode orders is unlikely to be significant at $\sim 1 \mathrm{~km}$ [8], thus it is concluded that this MPI is primarily due to imperfections in the free-space mux/demux. Furthermore, significant drifting of the mux/demux was observed, leading to cross-talk increasing with time, thus limiting the number of measurements that can be performed before re-alignment is needed (this being the primary reason only 3 rather than all 60 wavelength channels were measured). Therefore an improved mux/demux could significantly improve system performance.

Assuming that all $30 \mathrm{WDM}$ channels between $1550 \mathrm{~nm}$ and $1556 \mathrm{~nm}$ fail to carry the $|L|=6$ spin-orbit antialigned modes, and thus only support 10 modes, while the 30 longer wavelength channels support 12 modes, the demonstrated system potentially has a total aggregate capacity of $10.56 \mathrm{Tbit} / \mathrm{s}$ after $20 \% \mathrm{FEC}$ overhead has been deducted for all channels, which would be a 6-fold improvement compared to previous fiber-based OAM transmission systems.

\section{Summary and Conclusions}

12 OAM modes, each carrying a 10 GBd QPSK signal, have been transmitted through $1.2 \mathrm{~km}$ of fiber, without using MIMO. Furthermore WDM compatibility of the fiber and the mux/demux was demonstrated, by using 60 WDM channels with $25 \mathrm{GHz}$ spacing. To the best of our knowledge, this is the largest number of mode groups over which MIMO-free data has been transmitted over any length of optical fiber. The most significant limitations encountered in this demonstration were the difficulties and drifts associated with aligning 6 free-space optical arms with high precision. It is thus expected that developments in mux/demux technologies would significantly enhance the system capacity of MIMO-free systems using OAM fiber modes.

\section{Acknowledgements}

This research is supported, in part, by the DNRF Centre of Excellence SPOC (DNRF213), Innovations Fonden espace (0603-00514B), Office of Naval Research (ONR) MURI (N0014-13-1-0672); National Science Foundation (NSF) (DGE-1247312, ECCS-1310493).

\section{References}

[1] S. Ramachandran and P. Kristensen, "Optical vortices in fiber," Nanophotonics, 2, 455 (2013).

[2] N. Bozinovic, Y. Yue, Y. Ren, M. Tur, P. Kristensen, H. Huang, A. E. Willner, and S. Ramachandran, "Terabit-scale orbital angular momentum mode division multiplexing in fibers.," Science, 340, 1545 (2013).

[3] R.M. Nejad, K. Allahverdyan, P. Vaity, S. Amiralizadeh, C. Brunet, Y. Messaddeq, S. LaRochelle, and L. A. Rusch, "Mode Division Multiplexing Using Orbital Angular Momentum Modes Over 1.4-km Ring Core Fiber," J. Lightwave Technol, 34, 4252 (2016).

[4]P. Gregg, P. Kristensen, and S. Ramachandran, "Conservation of orbital angular momentum in air-core optical fibers," Optica, 2, 267 (2015).

[5]C. Brunet, P. Vaity, Y. Messaddeq, S. LaRochelle, and L. a Rusch, "Design, fabrication and validation of an OAM fiber supporting 36 states.," Opt. Express, 22, 26117 (2014).

[6] L. Marrucci, C. Manzo, and D. Paparo, "Optical Spin-to-Orbital Angular Momentum Conversion in Inhomogeneous Anisotropic Media," Phys. Rev. Lett., 96, 163905 (2006).

[7] L. Wang, P. Vaity, S. Chatigny, Y. Messaddeq, L.A. Rusch, and S. LaRochelle, "Orbital-Angular-Momentum Polarization Mode Dispersion in Optical Fibers," J. Lightwave Technol., 34, 1661 (2016).

[8] R. Maruyama, N. Kuwaki, S. Matsuo, and M. Ohashi, "Experimental Investigation of Relation Between Mode-Coupling and Fiber Characteristics in Few-Mode Fibers," OFC Conference 2015, M2C.1 